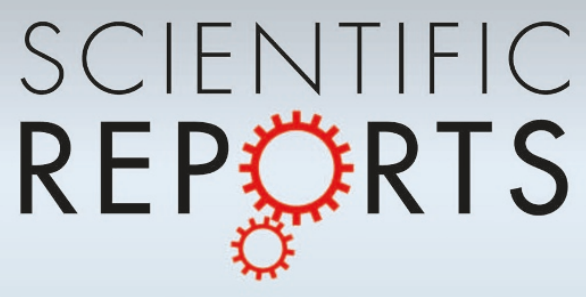

OPEN

SUBJECT AREAS:

TARGETED GENE REPAIR

TUMOUR ANGIOGENESIS

CANCER IMMUNOTHERAPY

DNA VACCINES

Received

26 June 2013

Accepted

11 November 2013

Published

29 November 2013

Correspondence and requests for materials should be addressed to

R.M. (morishit@cgt. med.osaka-u.ac.jp) or H.N. (nakagami@cgt. med.osaka-u.ac.jp)

\section{Development of novel DNA vaccine for VEGF in murine cancer model}

\author{
Mariko Kyutoku ${ }^{1,2}$, Hironori Nakagami ${ }^{2}$, Hiroshi Koriyama ${ }^{1,2}$, Hideki Tomioka ${ }^{1,2}$, Futoshi Nakagami i, , \\ Munehisa Shimamura' ${ }^{2}$, Hitomi Kurinami ${ }^{2}$, Pang Zhengda ${ }^{2,3}$, Dong Hyun Jo ${ }^{4,5}$, Jeong Hun Kim ${ }^{4,5,6}$, \\ Nobuyuki Takakura ${ }^{7,8} \&$ Ryuichi Morishita'
}

\begin{abstract}
'Department of Clinical Gene Therapy, Osaka University Graduate School of Medicine, ${ }^{2}$ Division of Vascular Medicine and Epigenetics, Department of Child Development, United Graduate School of Child Development, Osaka University, Kanazawa University, Hamamatsu University School of Medicine, Chiba University and Fukui University, ${ }^{3}$ Department of Geriatric Medicine, Osaka University Graduate School of Medicine, ${ }^{4}$ Fight against Angiogenesis-Related Blindness (FARB) Laboratory, Clinical Research Institute, Seoul National University Hospital, Seoul, Republic of Korea, ${ }^{5}$ Department of Biomedical Sciences, College of Medicine, Seoul National University, Seoul, Republic of Korea, 'Department of Ophthalmology, College of Medicine, Seoul National University, Seoul, Republic of Korea, ${ }^{7}$ Department of Signal Transduction, Research Institute for Microbial Diseases, Osaka University, Suita, Osaka, Japan, ${ }^{8}$ JST(Japan Science and Technology Agency), CREST, Tokyo, Japan.
\end{abstract}

We developed DNA vaccine for vascular endothelial growth factor (VEGF), which may provide the therapeutic option instead of anti-VEGF antibody, bevacizumab. Plasmid containing VEGF mini-gene was constructed in the insertion of B-cell epitope of Hepatitis B core protein [HBc-VEGF], which was an epitope carrier. High titer of anti-VEGF antibody was observed in $\mathrm{BALB} / \mathrm{c}$ mice which were intramuscularly immunized with HBc-VEGF by electropolator. In mice inoculated with colon 26 cells, tumor volume and microvessel density was decreased in HBc-VEGF with a significant prolonged survival. Co-treatment of purified IgG from immunized mice with HBc-VEGF showed in vitro neutralizing activity for VEGF-induced ERK phosphorylation and tube formation in cultured endothelial cells. Furthermore, intravitreally injection of this purified IgG reduced the neovessel formation in the mouse oxygen-induced retinopathy and laser-induced choroidal neovascularization models. These results first provided that DNA vaccine against VEGF possessed the anti-angiogenic effect, leading to prolonged survival in mouse cancer model. 
In this study, we selected mini-gene from the targeted antigen, VEGF, which are the residues related to VEGF-bevacizumab/ VEGFR binding, and developed DNA vaccine for VEGF using Hepatits B virus core $(\mathrm{HBc})$ system. $\mathrm{HBc}$ protein was used as an epitope carrier to enhance the immunogenicity as previously reported $^{16,17}$. The neutralizing activity of anti-VEGF antibody produced by immunization with DNA vaccine for VEGF was examined.

\section{Results}

Production of DNA vaccine for VEGF. To develop DNA vaccine for neutralizing VEGF, we selected the target antigen of VEGF, which showed the binding epitope of VEGF for the humanized anti-VEGF antibody, bevacizumab, and the binding region of VEGFR-1 or VEGFR-2 (Fig. 1a) ${ }^{18}$. The core candidate antigen was seven amino acids (MRIKPHQ; 7 a.a.) which overlapped the binding surface with bevacizumab, VEGFR-1 or VEGFR-2. Because only seven amino acids could be less immunogenic for vaccination, $\mathrm{HBc}$ protein was utilized as an epitope carrier protein which is able to self-assemble into icosahedral virus-like particles (VLPs) in heterologous expression systems. To confirm whether this DNA vaccine system would sufficiently induce anti-VEGF antibody production, BALB/c female mice were immunized with pcDNA3.1-HBc-mVEGF (7 a.a.) [HBcmVEGF (7 a.a.)], pcDNA3.1-HBc [HBc] or saline, respectively, by intramuscular administration using electroporator, three times every two weeks and additional booster after third immunization (Fig. 1a). As a result, high titer of anti-VEGF antibody was not observed in $\mathrm{HBc}-\mathrm{mVEGF}$ (7 a.a.) group compared to control ( $\mathrm{HBc}$ and saline) group (Fig. 1b). Because this 7 a.a. sequence might not be enough for B-cell epitope to induce anti-VEGF antibody, the long sequence was also designed as a candidate antigen, which covered the binding surface with bevacizumab, VEGFR-1 or VEGFR-2. We added 6 or 10 amino acids to core sequence, and created the target 13 -amino acid sequence (IMRIKPHQSQHIG; 13 a.a.) and 17-amino acid sequence (MQIMRIKPHQSQHIGEM; 17 a.a.), respectively. Then, pcDNA3.1mVEGF (13 a.a.) [HBc-mVEGF (13 a.a.)] and pcDNA3.1-mVEGF (17 a.a.) [HBc-mVEGF (17 a.a.)] were similarly constructed and vaccinated to mice. As shown in Fig. 1c, both antigens successfully induced the production of anti-VEGF antibody. Relatively high titer of anti-VEGF was observed In HBc-mVEGF (13 a.a.) group as compared to 17 a.a. and 7 a.a groups (Fig. 1c). Thus, we decided 13 a.a. (IMRIKPHQSQHIG) as the antigen of VEGF DNA vaccine using $\mathrm{HBc}$ system (SI. 1a and 1b). The functionality of expression vector of HBc-mVEGF (13 a.a.) was confirmed in transfected COS-7 cells. Each construct expressed mRNA of the expected length and protein of expected molecular mass (SI. 2a).

We confirmed the titer of anti-VEGF antibody at eighth week, four weeks after third immunization (Fig. 1d), and high titer was observed in HBc-mVEGF (13 a.a.) group (Fig. 1e, 1f). In the analysis of IgG subtypes, this immunization could lead to Th1-biased immune responses with predominant IgG2a and IgG2b production (Fig. 1e). We further confirmed whether the produced serum by immunization with HBc-mVEGF (13 a.a.) would recognize the recombinant mouse VEGF-A protein. The specific binding of immunized serum with both mouse and human was shown in western blot analysis, confirmed by using the commercial antibody against VEGF, VG-1 (Fig. 1f and SI. 2b).

Neutralizing activity of anti-VEGF antibody produced by VEGF DNA vaccination. To examine the neutralizing activity of serum from vaccinated mice, we purified mouse IgG of serum using by Protein G column as described in method section (SI. 1e). Treatment of immunized serum, but not control serum, significantly attenuated VEGF-A-induced ERK1/2 phosphorylation in HUVECs (Fig. $2 \mathrm{a}$ and SI. 3). In the tube formation of HUVECs in matrigel, in the presence of EBM- 2 with $0.4 \%$ FBS and $0.4 \%$ supplement, the addition of immunized serum also significantly attenuated the tube formation of HUVECs, as compared to the addition of control serum (Fig. 2b).

Anti-tumor effects in tumor-bearing mice with colon 26 carcinoma cells. Given the neutralizing activity of the immunized serum in vitro, we injected colon 26 cells on mouse back subcutaneously one week after last immunization (Fig. 3a). In this tumor-bearing model, mice perish from cancer cachexia contributed some mediators such as IL-6, IL- 1 and TNF $\alpha$ on day 14 to 20 after cancer cell implantation ${ }^{19,20}$. $\mathrm{HBc}-\mathrm{mVEGF}$ (13 a.a.) vaccination or $\mathrm{HBc}$ control was intramuscularly administred using electroporator, three times every two weeks and additional booster after third immunization, similarly. The mice vaccinated with $\mathrm{HBc}-\mathrm{mVEGF}$ showed a significant inhibition of primary tumor growth in early phase as compared to the mice of control group (Fig. 3b). In the microvessel density in tumor tissue, as the indication of effects of anti-angiogenic agents assessed by immunohistochemical staining for CD31, immunization with $\mathrm{HBc}$ mVEGF significantly decreased CD31-positive cells, as compared to control group in the subcutaneous colon 26 tumor model (Fig. 3c). Of importance, consistent with these results, DNA vaccine for VEGF significantly improved the survival in this cancer model through the VEGF-neutralizing activity (Fig. 3d).

In vivo anti-angiogenic effects of VEGF DNA vaccine on retinal and choroidal neovascularization. To further confirm in vivo neutralizing effect of purified mouse IgG of serum from immunized mice, we intravitreally injected purified mouse IgG into the eyes of mouse oxygen-induced retinopathy (OIR) and laser-induced choroidal neovascularization $(\mathrm{CNV})$ models. Each model represents the pathologic conditions mimicking retinal neovascularization and choroidal neovascularization, respectively. Both conditions are known to be mainly mediated by VEGF ${ }^{21}$. Interestingly, the purified mouse IgG effectively reduced the formation of vascular tufts and the number of vascular lumens in the mouse OIR model (Fig. 4a and SI. 3). Furthermore, the area of CNV demonstrated by isolectin B4 staining was also significantly decreased by the treatment with the purified mouse IgG of serum from mice previously immunized with $\mathrm{HBc}$ mVEGF (Fig. 4b). Particularly, anti-angiogenic and neutralizing activities of anti-VEGF antibody from the purified mouse IgG were comparable to those of commercialized monoclonal anti-VEGF antibody, bevacizumab (Fig. $4 \mathrm{a}$ and $4 \mathrm{~b}$ ).

\section{Discussion}

Tumor angiogenesis is a crucial process in tumor development. Among the angiogenic factors, VEGF-A is the major player both in physiological angiogenesis and in pathological angiogenesis. This recognition has led to VEGF-targeted approaches, the development of several VEGF-targeted agents including neutralizing antibodies to VEGF/VEGFR, soluble VEGFR/VEGFR hybrids and tyrosine kinase inhibitors with selectivity for $\mathrm{VEGFR}^{22}$. VEGFtargeted agents, administered either as single agents or in combination with chemotherapy, have been shown to benefit the patients with advanced-stage malignancies. The effects of VEGF-targeted therapy were mainly divided into two paradigms, the inhibition of new blood vessel growth and normalization of the tumor vasculature which may contribute the therapeutic effects ${ }^{23-26}$.

Vaccines for VEGF or its receptor have been reported to induce the CTL-mediating cellular immunity as well as antibody-mediating humoral immunity against tumor ${ }^{14,15,27}$. Because VEGF is produced from normal vascular or interstitial cells, and its receptors are widely expressed in endothelial cells, enhanced CTL-mediating cellular toxicity for long term may induce the side effect in terms of safety issue such as hypertension, myocardial and cerebrovascular infarction. Therefore, we designed epitope vaccine to activate humoral immunity, but not cellular immunity for VEGF. First, we examined the most appropriate epitope to either inhibit or neutralize the activity for 


\section{a.}
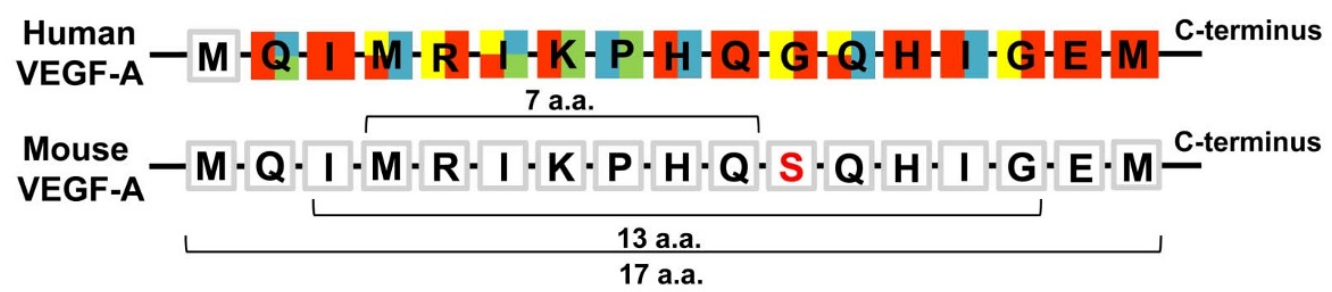

b.

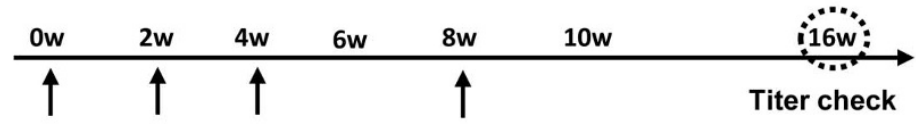

C.

\section{Total IgG}
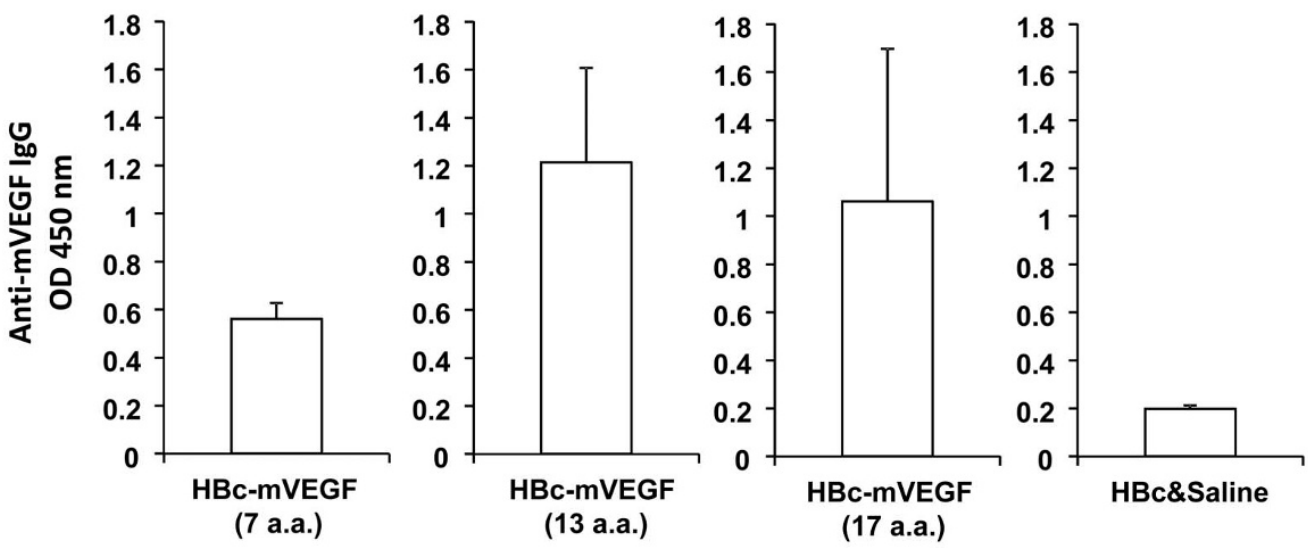

d.

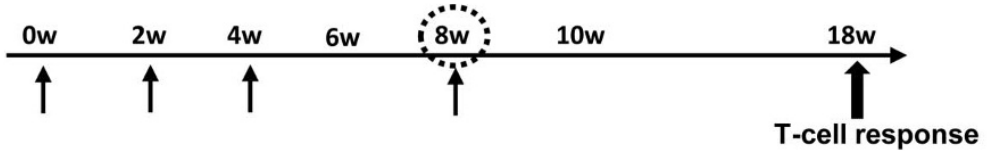

e.

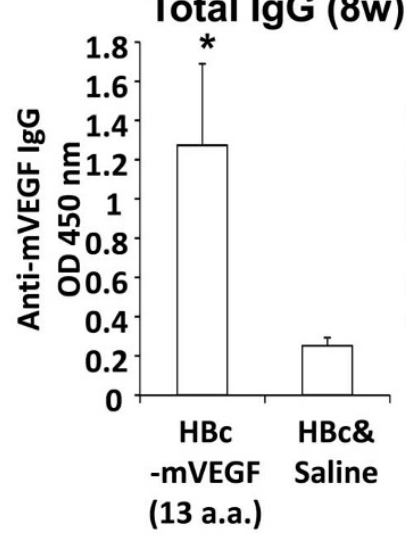

IgG subtype (8w)

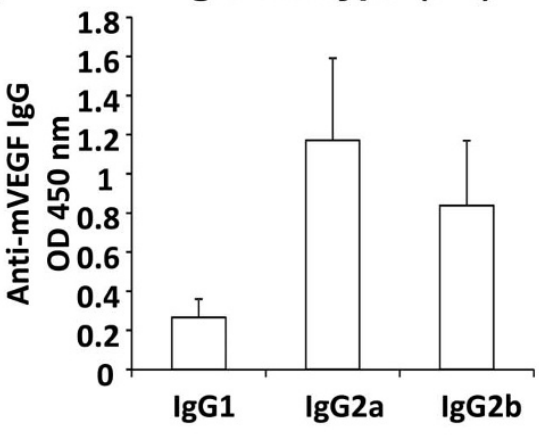

f.

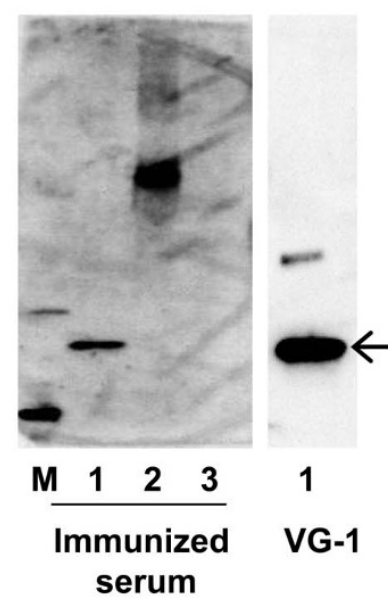

Figure 1 DNA vaccination for VEGF in BALB/c. (a) Framework residues at VEGF-bevacizumab interface. Red, residues in binding surface with bevacizumab; Yellow, especially important residues of Red; Blue, residues in binding surface with VEGFR-1; Green, residues in binding surface with VEGFR-2. Each residue is represented by single-letter codes. (b) and (d) Time course of DNA vaccination. Vaccination was initially performed using 6 week-old mice $(0 \mathrm{w})$, and subsequent vaccinations were given at $2(2 \mathrm{w}), 4$ or 16 weeks after first vaccination. (c) Titers of anti-VEGF antibodies at 16 weeks. Total IgG titers for VEGF were quantified in mouse sera (100 dilution) from mice immunized with HBc-mVEGF (7 a.a.), HBc-mVEGF (13 a.a.), HBcmVEGF (17 a.a.) or HBc, respectively. (e) Titers of anti-VEGF antibodies at 8 weeks. Total IgG titers for VEGF were increased only in mouse sera (100 dilution) from the HBc-mVEGF (13 a.a.) group (left panel). The IgG subtype distribution (IgG1, IgG2a or IgG2b) was also evaluated using subtypespecific IgG antibodies in mouse sera (100 dilution) from the HBc-VEGF (13 a.a.) group (right panel). (f) Specific binding of immunized serum to VEGF. Immunized serum used as primary antibody in western blot bound to not only BSA-conjugated mVEGF (13 a.a.) but recombinant mouse VEGF (rmVEGF). Loading samples were as follows; lane 1, recombinant mouse VEGF-A; lane 2, BSA-conjugated mVEGF (13 a.a.); lane 3, BSA-conjugated human Angiopoietin-2 peptide as negative protein. VG-1, commercial monoclonal antibody against VEGF, was used as positive antibody. Data were means \pm S.E.M. ${ }^{*} \mathrm{p}<0.05$ versus control ( $\mathrm{HBc}$ and Saline). 


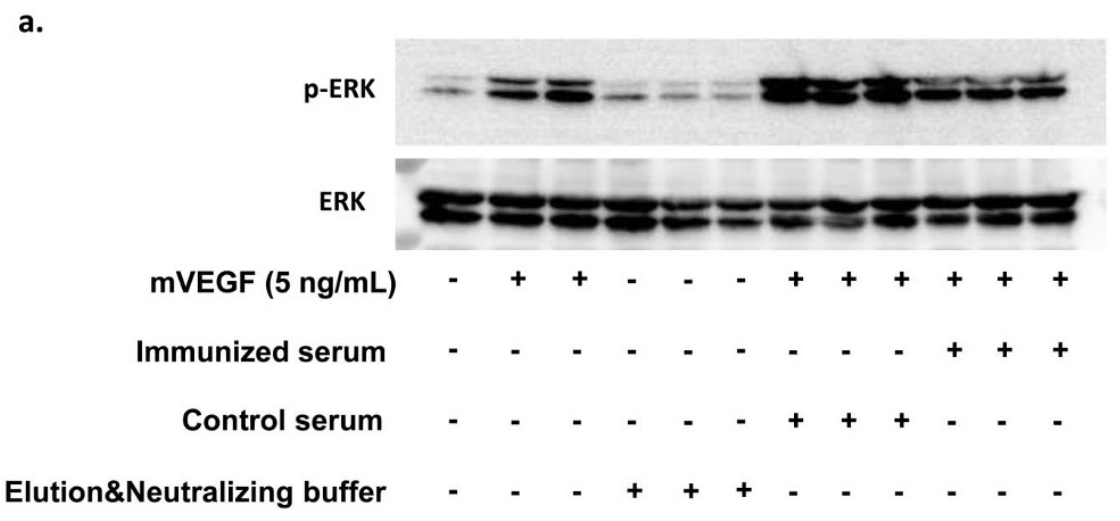

b.
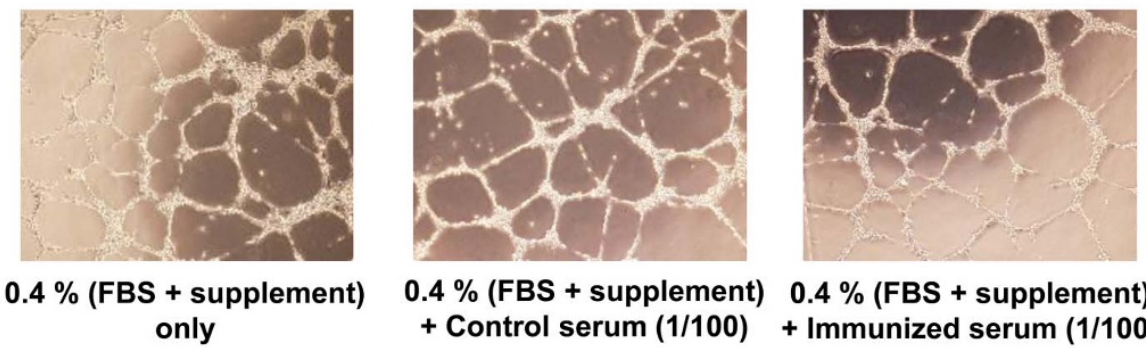

$0.4 \%$ (FBS + supplement) $\quad 0.4 \%$ (FBS + supplement)

+ Control serum (1/100) + Immunized serum (1/100)

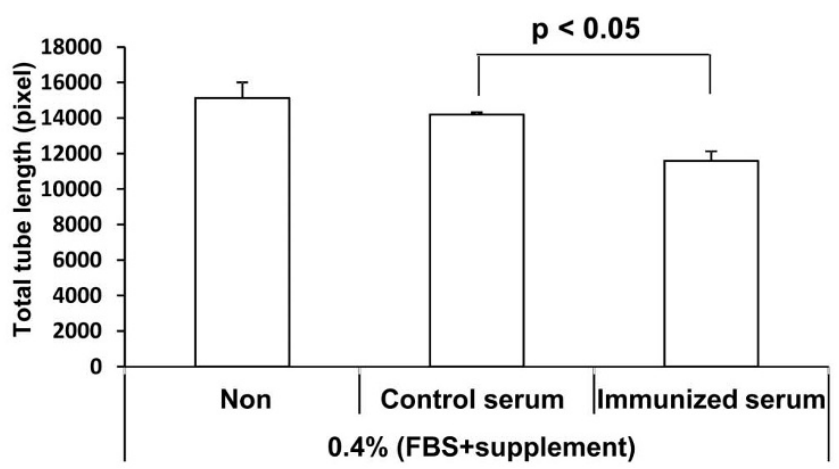

Figure $2 \mid$ Neutralizing activity of immunized serum in HUVECs. (a) Western blot analysis of cell lysates from HUVECs stimulated for 10 min with mVEGF at $5 \mathrm{ng} / \mathrm{mL}$ in the presence of immunized serum or control serum for p-ERK and total ERK under the same experimental condition. (b) Effects of immunized serum on VEGF-induced tube formation of HUVECs. HUVECs were plated on matrigel-coated plates at density of $1 \times 10^{5}$ cells/well and incubated in the presence of control serum or immunized serum. After 7 hours, capillary network were photographed and quantified. Representative endothelial tubes were shown. Magnification: $50 \times$. Data were means \pm S.E.M. of triplicates.

VEGF-A. The candidate epitopes were three peptides: 7, 13 and 17 amino acid peptide, which contained the binding epitope of VEGF for bevacizumab and the binding region of VEGFR-1 or VEGFR$2^{18,28}$. From the present study, we finally selected 13 a.a. sequence to produce high anti-VEGF antibody. To assess the validity of the epitope (13 a.a. in VEGF-A), we also performed T-cell proliferation assay and ELISpot assay. In immunized female BALB/c mice, T-cell proliferation assay showed that the stimulation with VEGF 13 a.a. did not induce the proliferation of splenocytes from immunized mice, but $\mathrm{rHBc}$ and $\mathrm{HBc}$ epitope induced the proliferation of splenocytes (SI. 4). Similarly, in the ELISpot assay, the stimulation with VEGF 13 a.a. induced the production of neither IFN- $\gamma$ nor IL-4, but stimulation with $\mathrm{rHBc}$ or $\mathrm{HBc}$ epitope did (SI. 5). In addition, the production of IFN- $\gamma$ by $\mathrm{rHBc}$ or HBc epitope was by far more than IL-4, which also indicated that this immunization could lead to Th1biased immune responses, along with the results of IgG subtype distribution. These data demonstrated that VEGF 13 a.a. did not induce $\mathrm{T}$-cell activation, but $\mathrm{HBc}$ did, suggesting that $\mathrm{HBc}$ worked as an epitope carrier.

In this study, we designed VEGF DNA vaccine for alternative of the present anti-VEGF antibody therapy. Especially, we focused on induction of neutralizing antibody, but not cytotoxic T-cell activation, as $\mathrm{HBc}$ has 'non-self helper $\mathrm{T}$ cell epitopes to elicit the potent $\mathrm{B}$ cell and $\mathrm{T}$-cell response in primates, and enhanced the immunogenicity of the epitope. Interestingly, anti-VEGF antibody induced by VEGF DNA vaccination showed in vitro neutralizing activity for ERK phosphorylation and tube formation in HUVECs and chemotactic activity in colon 26 cells. In vivo evidence also showed the inhibition of primary tumor growth through the inhibition of the number of CD31-positive vessels and the prolonged survival in tumor-bearing mice. From these results, combination with chemotherapy might be useful for VEGF DNA vaccination to enhance the therapeutic effects. Toward to the clinical trial, it might be considered for effective vaccination protocol, because our present study reflected 

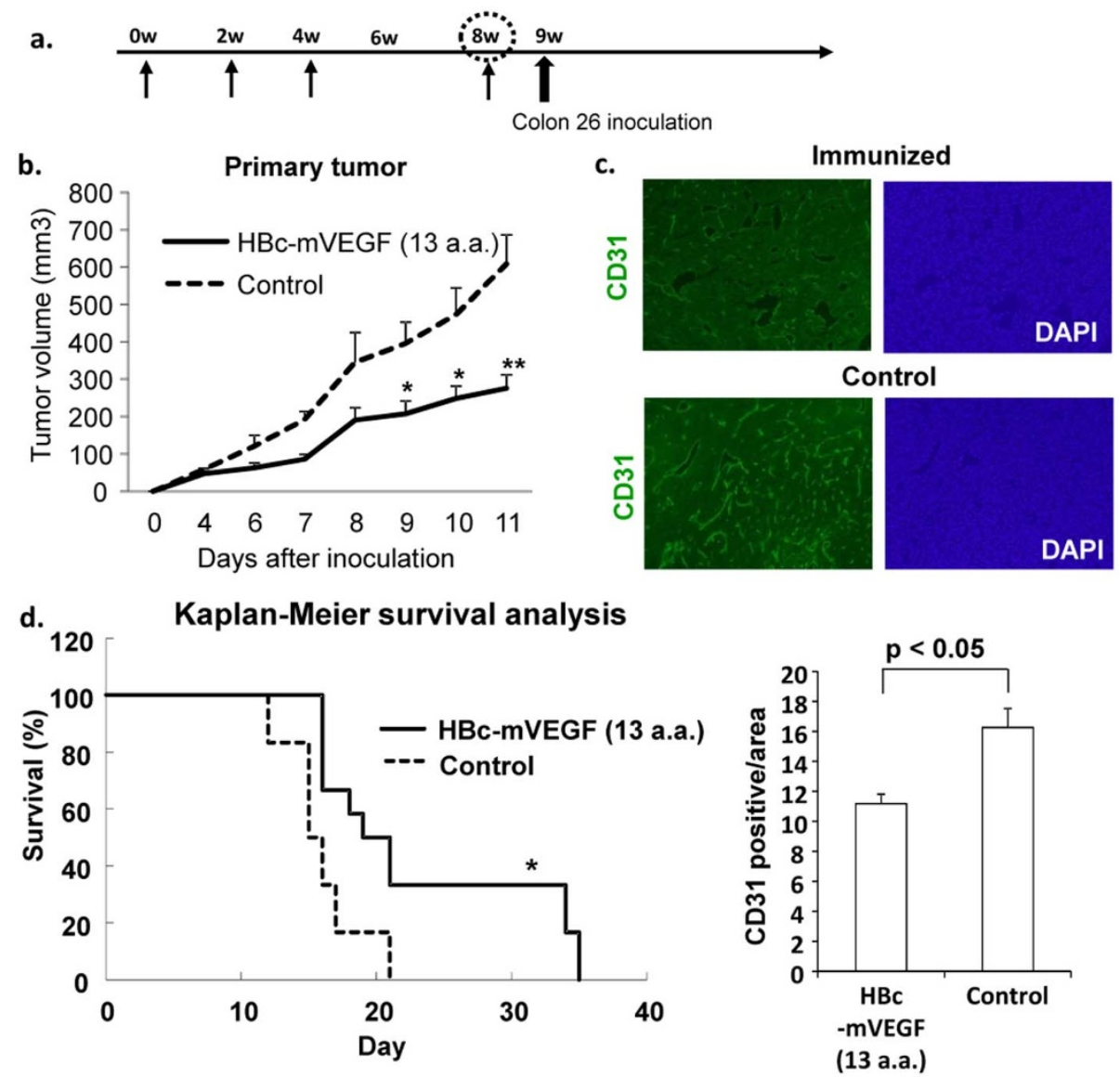

Figure 3 Effects of VEGF DNA vaccine in tumor-bearing mice. (a) Time course of DNA vaccination and tumor implantation. One week after last immunization, colon 26 cells were inoculated subcutaneously into mice. (b) Primary tumor volume. Tumor volume was measured by using caliper and the following formula: $0.5 \times$ (length) $\times(\text { width })^{2}$. HBc-mVEGF $(13$ a.a.) immunization inhibited the tumor growth compared with control group. (c) Immunohistochemistry staining of primary tumor tissue (CD31). Tumors were excised, sectioned, and stained with anti-CD31 antibody to visualize the blood vessels. The number of CD31-positive cells per area was counted. (d) Kaplan-Meier survival curve. ${ }^{*} \mathrm{p}<0.05$; ${ }^{* *} \mathrm{p}<0.01$ versus control.

the prevention model. It might be necessary to examine the therapeutic model to consider the clinical practice.

Overall, the present data first provided the potential activity to inhibit angiogenesis in cancer models, oxygen-induced retinopathy and laser-induced CNV models using DNA VEGF vaccine. DNA vaccine targeting mini-gene of VEGF might provide new therapeutic option in combination with chemotherapies or immunotherapies. We anticipate that the modification of this VEGF DNA vaccine will increase its potential clinical utility for the treatment of angiogenesisrelated diseases such as cancer.

\section{Methods}

Construction of HBc-mVEGF fusion gene expression vector. We used the plasmid pcDNA3.1 (pcDNA3.1/V5-His-TOPO, Invitrogen) containing the cytomegalovirus promoter. The constructs were verified by nucleotide sequencing, by mRNA expression on RT-PCR and by protein expression on Western blots following transient transfection into COS-7 cells (SI. 1c).

Animals and vaccination protocol. The experiments were approved by the Ethical Committee for Animal Experiments of the Osaka University Graduate School of Medicine. The mice had free access to water and food during the experimental periods. Five-week-old female BALB/c mice were purchased from CLEA Japan, Inc. and were acclimated for 1 week. Female BALB/c mice were vaccinated intramuscularly three times at 2-week intervals (6,8 and 10 weeks old) with $60 \mu \mathrm{L}$ of TE containing $120 \mu \mathrm{g}$ of plasmid DNA or saline using an electric pulse generator with a pair of stainless steel needles that were $10 \mathrm{~mm}$ in length and $0.3 \mathrm{~mm}$ in diameter with a fixed distance between them of $3 \mathrm{~mm}$ (NEPA GENE). The voltage remained constant at $70 \mathrm{~V}$ during the pulse duration. Three pulses at the indicated voltage followed by three more pulses of the opposite polarity were administered to each injection site at a rate of one pulse/s, with each pulse being $50 \mathrm{~ms}$ in duration. Four weeks after the third immunization (14 weeks old), an additional immunization was administered to the mice.

Measurement of anti-mVEGF antibody in serum. Eight or sixteen weeks after first immunization, serum was collected from the immunized mice of all groups. Serum levels of anti-VEGF antibodies in these mice were measured by ELISA. Briefly, ELISA plates were coated with $5 \mu \mathrm{g} / \mathrm{mL}$ VEGF 13 a.a. peptide conjugated BSA in carbonate buffer overnight at $4{ }^{\circ} \mathrm{C}$. Serial dilutions $(1: 100$ to $1: 312500)$ of serum samples from the immunized mice were added to the wells, and HRP-conjugated mouse IgG (whole IgG; GE Health care, UK, each subtype; Abcam, UK) was added. After four washes with PBST, 3,3',5,5' -tetramethylbenzidine (TMB, Sigma-Aldrich, USA) was added. Production of the blue reaction product was stopped by adding $0.5 \mathrm{~mol} / \mathrm{L}$ sulfuric acid, and the resulting end product (yellow) was read at $450 \mathrm{~nm}$.

T-cell proliferation assay and enzyme-Linked ImmunoSpot (ELISpot) assay. The T-cell proliferation assay was performed as previously reported. Cultured splenocytes from mice immunized with the HBc-mVEGF (13 a.a.) vaccine plasmid DNA either were not stimulated or stimulated with peptides containing the antigen sequence (mVEGF 13 a.a.; IMPIKPHQSQHIGE), recombinant $\mathrm{HBc}$ protein (rHBc), peptides containing the HBc CTL epitope, or phytohemagglutinin (PHA, $50 \mu \mathrm{g} / \mathrm{mL}$, as positive control). Syngeneic T cells (mouse splenocytes, $5 \times 10^{5}$ cells/well) were cultured with several stimulants at $37^{\circ} \mathrm{C}$ in $5 \% \mathrm{CO}_{2}$ for 40 hours. Furthermore, $1 \mu \mathrm{Ci}$ of $\left[{ }^{3} \mathrm{H}\right]$ thymidine (Perkin Elmer) was added to each well for 8 hours. The cells were harvested, and the $\left[{ }^{3} \mathrm{H}\right]$ thymidine uptake $(\mathrm{cpm})$ was determined using a MicroBeta 1450 Trilux scintillation counter (Wallac Oy). The stimulation index was expressed as the ratio of stimulated cells to non-stimulated cells.

The ELISpot assay was carried out using the Mouse IFN- $\gamma$ Development Module and the Mouse IL-4 Development Module for their respective targets (R\&D Systems, USA) according to the manufacturer's instructions.

IgG purification. Mouse serum was purified using by Protein $\mathrm{G}$ column according to the manufacturer's instructions (MAbTrap Kit; GE Healthcare, UK). Briefly, serum samples were adjusted to the composition of the binding buffer $(20 \mathrm{mM}$ sodium phosphate, $\mathrm{pH}$ 7.0). The Protein $\mathrm{G}$ column was washed out the ethanol preservative 
a.

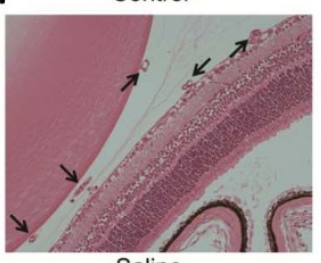

Saline

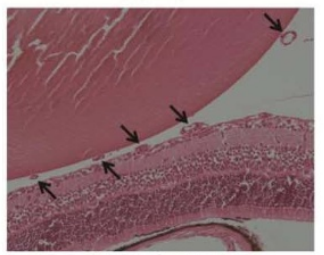

Bevacizumab

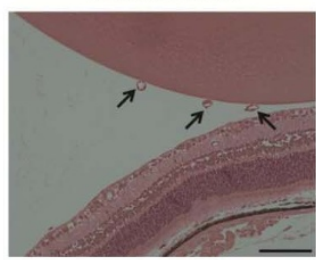

$\mathrm{HBc}$
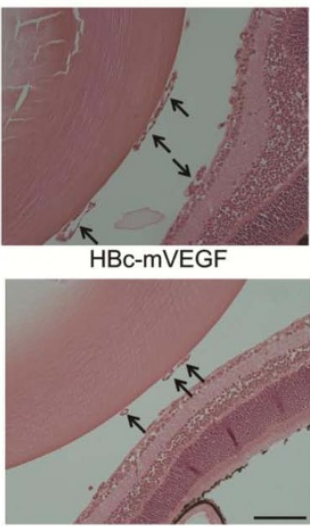

C.

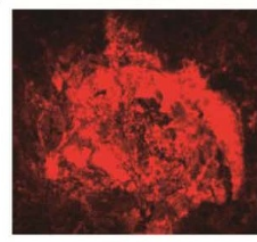

Saline

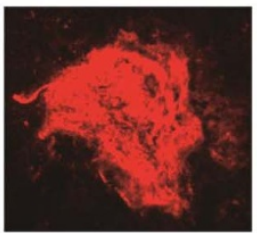

Bevacizumab

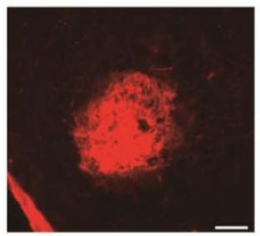

$\mathrm{HBc}$

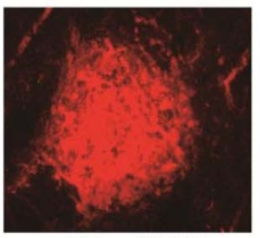

HBc-mVEGF
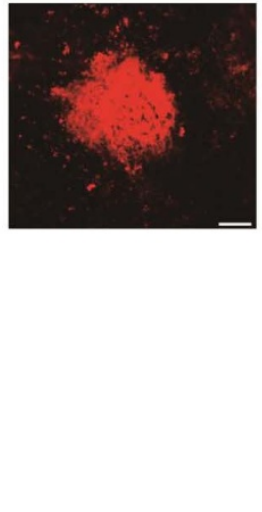

b.

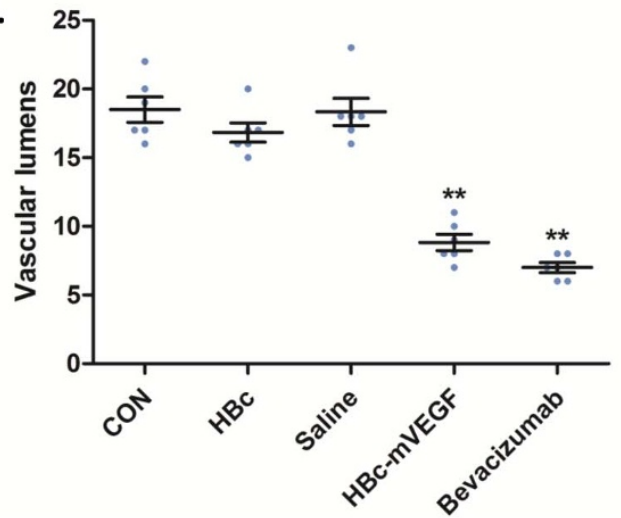

d.

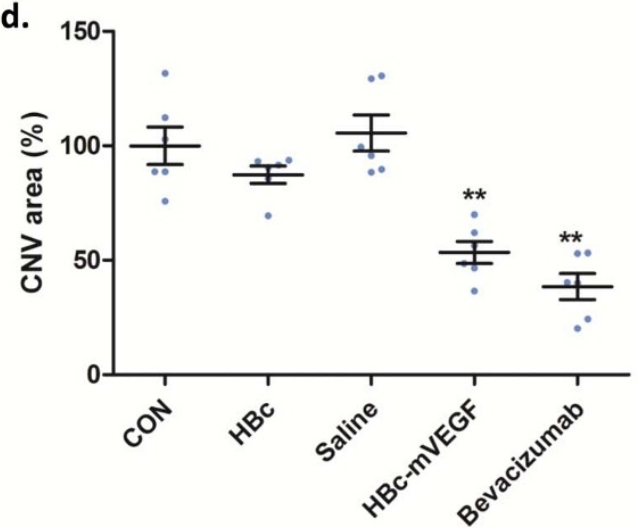

Figure $4 \mid$ Neutralizing activity of immunized serum in retinal and choroidal neovascularization. (a) Effects of immunized serum on retinal neovascularization in mouse OIR model. Purified IgG from immunized serum was intravitreally injected into the right eyes of mice at P14 and the enucleated eyes were prepared for further analyses at P17. For qualitative analyses, FITC-dextran (500 kDa) was intravenously perfused and the retinal tissues were prepared and flat-mounted. The images from each group was intentionally modified and combined to demonstrate the inhibitory effect of the purified IgG on the formation of vascular tufts. Quantitative analysis was performed by direct counting of vascular lumens on the vitreal side of the inner limiting membrane in at least 10 sections from each eye $(n=6)$. Data were means \pm S.E.M. CON, control; HBc, purified IgG from mice immunized with $\mathrm{HBc}$; Saline, purified IgG from mice immunized with saline; HBc-mVEGF; purified IgG from mice immunized with HBc-mVEGF. **p $<0.01$. Scale bar, $100 \mu \mathrm{m}$. (b) Effects of immunized serum on mouse laser-induced CNV model. CNV was induced by laser photocoagulation. Scale bar, $100 \mu \mathrm{m}$. Purified IgG from immunized serum was intravitreally injected into the right eyes of mice at 10 days after the photocoagulation and the enucleated eyes were prepared for further analyses after additional 4 days. Quantitative analysis was performed by measuring the CNV area in 4 burn sites from each eye $(n=$ 6). Data were means \pm S.E.M. CON, control; HBc, purified IgG from mice immunized with $\mathrm{HBc}$; Saline, purified IgG from mice immunized with saline; HBc-mVEGF; purified IgG from mice immunized with HBc-mVEGF. ${ }^{* *} \mathrm{p}<0.01$. Scale bar, $100 \mu \mathrm{m}$.

with distilled water and equilibrated with binding buffer. The diluted serum was applied and the column was wash with binding buffer until no material appears in the effluent. After that, IgG fraction was eluted by elution buffer $(0.1 \mathrm{M}$ glycine- $\mathrm{HCl}, \mathrm{pH}$ 2.7) and concentrated by centrifugal filter (Amicon Ultra; Millipore, USA).

Tube formation assay. The tube formation assay using Matrigel (Becton-Dickinson, USA) was performed according to manufacturer's instructions. Briefly, matrigel was used to coat the wells of 24 -well plates $(0.25 \mathrm{~mL}$ per well $)$ and was left to polymerize at $37^{\circ} \mathrm{C}$ for 1 hour. After polymerization, HUVECs (100,000 cells) suspended in $0.3 \mathrm{ml}$ of EBM-2 (containing 20\% EGM-2 kit, $0.4 \%$ FBS and $20 \%$ supplement) with control serum or immunized serum, were added to each well. After 6 hours, the wells were photographed at 50-fold magnification in five randomized fields (Olympus) and the number of their tubular networks was counted by using ImageJ.

Mouse tumor model. Colon 26 parental colon carcinoma cell line was generously gifted by the laboratory of Dr. Nobuyuki Takakura, and cells were cultured in
RPMI1640 medium containing 10\% FBS. $1 \times 10^{6}$ cells of colon 26 in $100 \mu \mathrm{L}$ PBS were inoculated subcutaneously into BALB/c mice in the right side of back $[\mathrm{n}=12$ for $\mathrm{HBc}$ mVEGF (13 a.a.) group, $n=3$ for $\mathrm{HBc}$ group or $\mathrm{n}=3$ for saline group]. The tumor volumes were calculated in $\mathrm{mm}^{3}$ as $0.5 \times($ length $) \times(\text { width })^{2}$. Survival was analyzed by the Kaplan-Meier method and compared between groups by use of the log-rank test.

Immunohistochemistry. Tumors were removed at 12 days after s.c. inoculation of colon 26 , fixed in $4 \%$ paraformaldehyde overnight, and equilibrated in phosphate buffer (PB) containing 15\% sucrose for 2 days, and in $\mathrm{PB} / 30 \%$ sucrose for 2 days. The samples were embedded for rapid freezing. Cross sections were laid on slides and stained; some sections were frozen at $-80^{\circ} \mathrm{C}$. For staining of microvessels, tissue sections air-dried at room temperature for $30 \mathrm{~min}$, were blocked with $3 \%$ normal goat serum/PBST for $30 \mathrm{~min}$ and incubated overnight at $4{ }^{\circ} \mathrm{C}$ with anti-mouse CD31 antibody (BD PharMingen, USA). Sections then were incubated with Alexa 488conjugated anti-rat IgG (Molecular Probes, USA) and counterstained. The microvessel density was evaluated by counting CD31-positive vessels using 
fluorescence microscope (Olympus, Japan) at 400-fold magnification in randomly selected fields at the periphery of each section.

Oxygen induced retinopathy (OIR). OIR was induced in newborn mice as previously described by Smith et al. and modified by our group ${ }^{29,30}$. Briefly, newborn mice were exposed to hyperoxia $\left(75 \pm 0.5 \% \mathrm{O}_{2}\right)$ from $\mathrm{P} 7$ to $\mathrm{P} 12$ and returned to normoxia condition. At P14, we intravitreally injected $1 \mu \mathrm{l}$ of PBS or purified mouse IgG of serum from mice immunized with $\mathrm{HBc}-\mathrm{mVEGF}, \mathrm{HBc}$ or saline into the right eyes of mice ( $n=6$ per group). At $\mathrm{P} 17$, mice were sacrificed with $\mathrm{CO}_{2}$ gas inhalation and the eyes were enucleated. The enucleated eyes were fixed with $4 \%$ paraformaldehyde and processed for further analyses. For qualitative analyses, fluorescein isothiocyanate dextran (500 kDa; Sigma-Aldrich) was intravenously injected 1 hour before the sacrifice. From the enucleated eyes, the retinal tissues were prepared, flat-mounted, and observed via the fluorescence microscope (BX50; Olympus). For quantitative analyses, the enucleated eyes were prepared for paraffin sections. Quantitative analysis was performed by direct counting of vascular lumens on the vitreal side of the inner limiting membrane in at least 10 sections from each eye ( $n=6$ per group).

Laser-induced CNV. CNV lesions were induced in the right eyes of mice by laser photocoagulation as previously described ${ }^{31}$. Briefly, 6-week-old male C57BL/6 mice were anesthesized and the pupils were dilated with $1 \%$ tropicamide (Alcon Laboratories, USA). Laser photocoagulation was performed to each 3, 6, 9, and 12 o'clock position of 2 disc diamters from the optic disc by using the indirect head set delivery system (Oculight; Iridex, USA) and a handheld +78 diopter lens. On the 10th day after the laser photocoagulation, we intravitreally injected $1 \mu \mathrm{l}$ of PBS or purified mouse IgG of serum from mice immunized with HBc-mVEGF, HBc or saline into the right eyes of mice ( $n=6$ per group). After additional 4 days, the mice were sacrificed with $\mathrm{CO}_{2}$ gas inhalation and the eyes were enucleated to investigate the antiangiogenic effect of tested molecules on choroidal neovascularization. For quantitative analyses, the enucleated eyes were prepared for immunofluorescence staining. After 1 hour fixation with $4 \%$ paraformaldehyde, the retinal pigment epitheium (RPE)-choroidsclera complex was prepared. The RPE-choroid-sclera complex was washed with cold buffer (1\% Triton X-100, $0.1 \mathrm{mM} \mathrm{CaCl}_{2} \cdot 2 \mathrm{H}_{2} \mathrm{O}, 0.1 \mathrm{mM} \mathrm{MgCl}$, and $0.1 \mathrm{mM}$ $\mathrm{MnCl}_{2} \cdot 4 \mathrm{H}_{2} \mathrm{O}$ ) in $\mathrm{pH} 6.8$ phosphate-buffered saline (PBS), treated with $1: 10$ Isolectin GS-IB4 conjugated with Alexa Fluor 568 (Invitrogen, USA) overnight, and washed 3 times with PBS. Then, flat-mounted RPE-choroid-sclera complex was evaluated with the fluorescence microscopy (BX50; Olympus).

Statistical analysis. Results were subjected to statistical analysis. Survival curves were analyzed using Kaplan-Meier method with log-rank test. Student's $t$ test was two-tail and unpaired.

Detailed information is described in the supplementary information.

1. Batchelor, T. T. et al. AZD2171, a pan-VEGF receptor tyrosine kinase inhibitor, normalizes tumor vasculature and alleviates edema in glioblastoma patients. Cancer Cell 11, 83-95 (2007).

2. Kane, R. C. et al. Sorafenib for the treatment of advanced renal cell carcinoma. Clin Cancer Res 12, 7271-7278 (2006)

3. Teng, L. S. et al. Clinical applications of VEGF-trap (aflibercept) in cancer treatment. J Chin Med Assoc 73, 449-456 (2010).

4. Hurwitz, H. et al. Bevacizumab plus irinotecan, fluorouracil, and leucovorin for metastatic colorectal cancer. New Engl J Med 350, 2335-2342 (2004).

5. Sandler, A. et al. Paclitaxel-carboplatin Alone or with Bevacizumab for Nonsmall-Cell Lung Cancer. New Engl J Med 355, 2542-2550 (2006).

6. Miller, K. et al. Paclitaxel plus bevacizumab versus paclitaxel alone for metastatic breast cancer. New Engl J Med 357, 2666-2676 (2007).

7. Friedman, H. S. et al. Bevacizumab alone and in combination with irinotecan in recurrent glioblastoma. J Clin Oncol 27, 4733-4740 (2009).

8. Rini, B. I. et al. Clinical response to therapy targeted at vascular endothelial growth factor in metastatic renal cell carcinoma: impact of patient characteristics and Von Hippel-Lindau gene status. BJU Inter 98, 756-762 (2006).

9. Saltz, L. B. et al. Bevacizumab in combination with oxaliplatin-based chemotherapy as first-line therapy in metastatic colorectal cancer: a randomized phase III study. J Clin Oncol 26, 2013-2019 (2008).

10. Giantonio, B. J. et al. Bevacizumab in combination with oxaliplatin, fluorouracil, and leucovorin (FOLFOX4) for previously treated metastatic colorectal cancer: results from the Eastern Cooperative Oncology Group Study E3200. J Clin Oncol 25, 1539-1544 (2007).

11. Holash, J. et al. VEGF-Trap: a VEGF blocker with potent antitumor effects. Proc Natl Acad Sci USA 99, 11393-11398 (2002).

12. Lockhart, A. C. et al. Phase I Study of Intravenous Vascular Endothelial Growth Factor Trap, Aflibercept, in Patients With Advanced Solid Tumors. J Clin Oncol 28, 207-214 (2010).
13. Gomez-Manzano, C. et al. VEGF Trap induces antiglioma effect at different stages of disease. Neuro-Oncology 10, 940-945 (2008).

14. Niethammer, A. G. et al. A DNA vaccine against VEGF receptor 2 prevents effective angiogenesis and inhibits tumor growth. Nat Med 8, 1369-1375 (2002).

15. Xie, K. et al. Anti-tumor effects of a human VEGFR-2-based DNA vaccine in mouse models. Genet Vac Ther 7, 10 (2009).

16. Pumpens, P. \& Grens, E. HBV core particles as a carrier for B cell/T cell epitopes. Intervirology 44, 98-114 (2001).

17. Mao, D. et al. Intramuscular immunization with a DNA vaccine encoding a 26 amino acid CETP epitope displayed by HBc protein and containing CpG DNA inhibits atherosclerosis in a rabbit model of atherosclerosis. Vaccine 24, 4942-4950 (2006).

18. Muller, Y. A. et al. VEGF and the Fab fragment of a humanized neutralizing antibody: crystal structure of the complex at $2.4 \overline{\bar{T}} \bullet$ resolution and mutational analysis of the interface. Structure 6, 1153-1167 (1998).

19. Strassmann, G., Jacob, C. O., Evans, R., Beall, D. \& Fong, M. Mechanisms of experimental cancer cachexia. Interaction between mononuclear phagocytes and colon-26 carcinoma and its relevance to IL-6-mediated cancer cachexia. J Immunol 148, 3674-3678 (1992).

20. Pedersen, A. E., Buus, S. \& Claesson, M. H. Treatment of transplanted CT26 tumour with dendritic cell vaccine in combination with blockade of vascular endothelial growth factor receptor 2 and CTLA-4. Cancer Lett 235, 229-238 (2006).

21. Grossniklaus, H. E., Kang, S. J. \& Berglin, L. Animal models of choroidal and retinal neovascularization. Prog Retinal Eye Res 29, 500 (2010).

22. Casanovas, O., Hicklin, D. J., Bergers, G. \& Hanahan, D. Drug resistance by evasion of antiangiogenic targeting of VEGF signaling in late-stage pancreatic islet tumors. Cancer Cell 8, 299-309 (2005).

23. Kim, E. S. et al. Potent VEGF blockade causes regression of coopted vessels in a model of neuroblastoma. Proc Natl Acad Sci USA 99, 11399-11404 (2002).

24. Inai, T. et al. Inhibition of vascular endothelial growth factor (VEGF) signaling in cancer causes loss of endothelial fenestrations, regression of tumor vessels, and appearance of basement membrane ghosts. Am J Pathol 165, 35-52 (2004).

25. Jain, R. K. Normalization of tumor vasculature: an emerging concept in antiangiogenic therapy. Science 307, 58-62 (2005)

26. Carmeliet, P. \& Jain, R. K. Principles and mechanisms of vessel normalization for cancer and other angiogenic diseases. Nat Rev Drug Discov 10, 417-427 (2011).

27. Dong, J. et al. A comparative study of gene vaccines encoding different extracellular domains of the vascular endothelial growth factor receptor 2 in the mouse model of colon adenocarcinoma CT-26. Cancer Biol Ther 7, 502-509 (2008).

28. Baca, M., Presta, L. G., O'Connor, S. J. \& Wells, J. A. Antibody humanization using monovalent phage display. J Biol Chem 272, 10678-10684 (1997).

29. Smith, L. et al. Oxygen-induced retinopathy in the mouse. Invest Ophth Vis Sci 35 , 101-111 (1994).

30. Kim, J., Yu, Y., Shin, J., Lee, H. \& Kim, K. Deguelin inhibits retinal neovascularization by down-regulation of HIF1- $\alpha$ in oxygen-induced retinopathy. J Cell Mol Med 12, 2407-2415 (2008).

31. Kim, J. H. et al. Antiangiogenic effect of deguelin on choroidal neovascularization. J Pharmacol Exp Ther 324, 643-647 (2008).

\section{Acknowledgments}

The authors appreciate Dr. Nobuyuki Takakura's great advice and discussion. We also thank Ms. Hizuki Hamada and Ms. Yoko Horiguchi for their technical assistance and Ms. Satoe Kitabata for her help in office procedures.

\section{Author contributions}

M.K., H.N., N.T. and R.M. designed research; M.K., H.K., H.T., F.N., M.S., H.K. and P.Z. performed the experiments and prepared Figure 1-3. D.H.J. and J.H.K. prepared Figure 4. M.K. and H.N. wrote the paper. All authors discussed and agreed on the results.

\section{Additional information}

Supplementary information accompanies this paper at http://www.nature.com/ scientificreports

Competing financial interests: The authors declare no competing financial interests.

How to cite this article: Kyutoku, M. et al. Development of novel DNA vaccine for VEGF in murine cancer model. Sci. Rep. 3, 3380; DOI:10.1038/srep03380 (2013).

This work is licensed under a Creative Commons Attribution 3.0 Unported license. To view a copy of this license, visit http://creativecommons.org/licenses/by/3.0 